\title{
Neumomediastino espontáneo: revisión de tema
}

\section{Spontaneous Pneumomediastinum: A Review}

Fecha de recepción: 12 Noviembre 2016 | Fecha de aprobación: 31 Mayo 2017

\author{
María Piedad Londoño \\ Pontificia Universidad Javeriana, Colombia \\ Lina María Ávila Arenas \\ Pontificia Universidad Javeriana, Colombia
}

a Autora de correspondencia. Correo electrónico: linavilamd@gmail.com

Cómo citar: Londoño MP, Ávila Arenas LM. Neumomediastino espontáneo: revisión de tema. Univ Med. 2017;58(4):1-7. doi: https://doi.org/10. 11144/Javeriana.umed58-4.neum

\begin{abstract}
RESUMEN
El neumomediastino espontáneo se define como la presencia de aire en el mediastino sin que tenga relación con patología traumática, procedimientos quirúrgicos o ventilación mecánica. Se considera una patología benigna y de rara presentación con una sintomatología que puede orientar a otros diagnósticos relacionados con patologías cardiovasculares, digestivas o respiratorias. Con una baja incidencia, la literatura reporta la presencia de un caso por cada 14.000 consultas al servicio de urgencias. Es más frecuente en adultos jóvenes, de sexo masculino, que manifiestan dolor torácico y disnea con otros hallazgos físicos relacionados que hacen sospechar el diagnóstico como la evidencia de enfisema subcutáneo. El artículo presenta una revisión de tema sobre neumomediastino espontáneo, patología poco frecuente, pero de importancia clínica en el diagnóstico diferencial del dolor torácico.

Palabras clave

neumomediastino espontáneo; mediastino.
\end{abstract}

\begin{abstract}
Spontaneous pneumomediastinum is defined as the presence of air in the mediastinum that's not directly related to a traumatic pathology, surgical procedures or mechanical ventilation. It is considered a benign pathology which rarely occurs with symptoms that can lead to other diagnosis related to cardiovascular, gastrointestinal or respiratory pathologies. It also has a low incidence according to literature, with reports of only one case for every 14,000 patients that visit emergency care services, having a higher incidence on young male adults that manifest thoracic pain and dyspnea, with additional related physical findings similar to a subcutaneous emphysema. This is a revision concerning spontaneous pneumomediastinum, a low incidence pathology with a clinical importance in the differential diagnosis of thoracic pain.

Keywords

pneumomediastinum spontaneous; mediastinum.
\end{abstract}

\section{Introducción}

La cavidad mediastinal es el área definida por la cavidad pleural, de forma lateral; la entrada del tórax, en la parte 
superior; la columna torácica, en la región posterior; el esternón, en la región anterior, y el diafragma, en la región inferior. El neumomediastino se define como la presencia de aire libre en el mediastino [1], que puede ser dividida en dos grupos: el primero, el neumomediastino con una causa clara desencadenante como producto de traumatismos, infecciones intratorácicas por bacterias productoras de gas o ruptura esofágica secundaria a episodios eméticos o exploraciones endoscópicas (neumomediastino secundario) [2, 3]. El segundo, neumomediastino espontáneo o síndrome de Hamman, en el cual no existe una causa obvia por la que se ocasione la presencia de aire intersticial en el mediastino, no existe una relación con patologías traumáticas, cirugías u otros procedimientos médicos, ni antecedente de ventilación mecánica asociada (neumomediastino primario) $[2,3,4,5,6,7$, 8]. El diagnóstico se basa en la sospecha clínica, dado que su presentación puede orientar hacia otros diagnósticos más comunes relacionados con patologías cardiopulmonares o digestivas. Por eso, se presenta una revisión de tema sobre neumomediastino espontáneo, su presentación, diagnóstico y orientación terapéutica.

\section{Discusión}

El neumomediastino fue reportado por primera vez por René Laennec, en 1827; posteriormente, en 1939, Louis Hamman describió el neumomediastino espontáneo o síndrome de Hamman en una serie de casos; no obstante, fue Macklin quien desde 1939 describió su fisiopatología, explicada a través de la ruptura de los alvéolos, que permiten que el aire, a través del espacio intersticial del árbol traqueobronquial, realice una disección de estos planos y genere el enfisema en el mediastino. Tal teoría fue confirmada en 1944 y permanece vigente hasta hoy $[7,8]$.

El neumomediastino se define como la presencia de aire libre en el mediastino [1]. Este puede ser primario o secundario, si hay o no una causa clara desencadenante $[2,4,6,7,9$,
10]. La incidencia del síndrome de Hamman ha sido reportada en una de cada 44.000 visitas al servicio de urgencias $[11,12]$, con una relación hombre mujer 8:1 que incluso puede llegar hasta 14:3 [7, 12, 13]. Para la población pediátrica se muestra una incidencia similar cercana a un caso por cada 30.000 ingresos al servicio de urgencias, con una edad promedio de presentación 17,5 a 25 años, reportada en el estudio realizado por Sung Hoon Kim y colaboradores, en la ciudad de Changwon, entre marzo del 2005 y junio del 2013 $[14,15,16]$. El curso clínico es habitualmente benigno y autolimitado; pero existen casos con evolución más agresiva y tórpida, secundarios al desarrollo de complicaciones graves como neumomediastino a tensión y neumotórax a tensión concomitante [5].

En la literatura sobre el tema se han descrito factores desencadenantes comunes para la presentación de un neumomediastino espontáneo, como las maniobras de Valsalva, crisis asmáticas (21\%), episodios eméticos (36 $\%)$, tos (7\%), esfuerzo al realizar la deposición, durante la realización de pruebas de función pulmonar y la inhalación de sustancias de abuso como cocaína o consumo de anfetaminas [2, 7, $11,17,18]$.

El neumomediastino espontáneo es una condición poco común y generalmente autolimitada; a menudo, se presenta en hombres jóvenes con la aparición súbita de dos síntomas principales: dolor torácico (75\%) y disnea (49 \%) $[7,11,17]$. El dolor torácico se irradia típicamente al dorso y al cuello (36\%), se intensifica con la deglución, la posición de sedestación y con los movimientos respiratorios, disfonía, disfagia (18\%) y edema en el cuello, síntomas que pueden hacer sospechar otras patologías más comunes y que dejan de lado el diagnóstico de neumomediastino [4].

En el examen físico, el hallazgo más encontrado es la presencia de enfisema subcutáneo alrededor del cuello y sobre los hombros, con una incidencia de presentación de entre el $40 \%$ y el $100 \%$. Además, es posible encontrar el signo de Hamman, que se refiere a "crujidos o burbujas" que se presentan con cada latido del corazón a la auscultación del tórax, con 
una prevalencia reportada de $12 \%$ al $18 \%$ [7, $11]$.

En un estudio realizado en la Clínica Mayo en Rochester, Minnesota, se buscaron los casos de síndrome de Hamman en el periodo comprendido entre el 1 de julio de 1997 y el 30 de junio del 2008, con un total de 62 pacientes adultos distribuidos así: 41 hombres y 21 mujeres con una media de edad de 30 años. El dolor torácico fue el síntoma predominante en 39 pacientes (63\%). Otros síntomas menos comunes fueron disnea, tos, dolor cervical, disfagia y disfonía. En el examen físico, 28 pacientes (45\%) reportaron enfisema subcutáneo, y ninguno, el signo de Hamman [4]. En este estudio, 47 pacientes (76\%) fueron hospitalizados con un promedio de estancia hospitalaria de 2,5 días; de estos, 23 pacientes (49\%) fueron ingresados por neumomediastino espontáneo, mientras que los otros 24 (51 $\%)$ se hospitalizaron por otras razones, como cetoacidosis diabética, complicaciones postransplante, absceso abdominal, neumonitis por hipersensibilidad, asma, neumonía y otras.

En ocasiones, el diagnóstico diferencial puede ser difícil, dada la presencia de síntomas similares entre las diferentes patologías, entre ellos [9, 18]: el síndrome de Boerhaave, que consiste en la ruptura de la pared esofágica posterior a episodios eméticos severos y se caracteriza por la presencia de dolor torácico severo; enfisema subcutáneo; mediastinitis; fiebre e hipotensión; síndrome coronario agudo; pericarditis; neumonía; neumotórax, y tromboembolismo pulmonar.

\section{Fisiopatología}

La patogénesis del neumomediastino espontáneo fue descrita por primera vez por Macklin en un modelo animal experimental (efecto de Macklin), quien evidenció que la hiperinflación alveolar, secundaria a un aumento de la presión intratorácica, causa un aumento agudo y súbito de la presión intraalveolar con la consecuente fuga de aire desde los espacios alveolares al intersticio, seguido por la migración proximal del aire hacia el hilio y el mediastino cercano a la vasculatura pulmonar. Una vez el aire alcanza el mediastino, se puede desplazar a lo largo de los planos tisulares alcanzando el cuello, la cara, el abdomen e, incluso, las extremidades [2, 4, 11]. Cuando el aire se acumula en el espacio pericárdico parietal anterior y recubre la pared torácica, produce el clásico signo de Hamman.

El neumomediastino espontáneo se ha visto relacionado con una gran variedad de patologías pulmonares que predisponen a los pacientes al desarrollo de esta entidad, incluidas enfisema, asma, enfermedad pulmonar intersticial $y$ bronquiectasias.

\section{Diagnóstico}

Teniendo en cuenta que la mayoría de los pacientes con neumomediastino espontáneo se presenta en el servicio de urgencias con dolor torácico como síntoma principal, debe existir la sospecha para llevar a cabo una correcta evaluación y orientación diagnóstica y terapéutica. Posterior a la realización de un abordaje integral en la historia clínica evaluando las características clínicas del dolor, síntomas asociados, desencadenantes, antecedentes personales del paciente y la realización de un juicioso examen físico, se solicitan métodos diagnósticos, como radiografía de tórax, que pueden llegar a identificar el neumomediastino espontáneo en el $90 \%$ de los casos $[4,7,19]$. Sin embargo, se estima que hasta un $30 \%$ o más de los pacientes son dados de alta sin diagnóstico [7, 8].

En el examen radiológico de tórax es posible encontrar diferentes signos que sugieren la presencia de neumomediastino, como el signo de la vela del timo, en el cual hay suficiente cantidad de aire que hace que el timo se eleve (más común en la población pediátrica) [7]; el signo del anillo en la proyección lateral, que corresponde a la presencia de aire alrededor de la arteria pulmonar o en los bronquios fuente, líneas de aire en el mediastino superior, una silueta cardiaca prominente o enfisema subcutáneo en los hombros y cuello; el signo de la doble pared 
bronquial; el signo del diafragma continuo; el signo de neumopericardio (presencia de aire entre el esternón y el pericardio anterior), y el signo de la $\mathrm{V}$ de Naclerio (aire en el margen lateral de la aorta descendente). Sin embargo, hasta el $30 \%$ de los pacientes puede presentarse con un radiografía de tórax normal [7, 11, 14].

La tomografía de tórax es otra herramienta diagnóstica que, a diferencia de la radiografía, detecta pequeñas cantidades de aire en el mediastino; así mismo, en algunas oportunidades puede revelar la causa subyacente, por lo que es considerada el método diagnóstico estándar para esta patología [11, 17]. Otros métodos diagnósticos empleados, como el esofagograma, la esofagoscopia y la broncoscopia deben ser realizados para evaluar la presencia de lesiones esofágicas o en el árbol traqueo bronquial.

Los hallazgos de laboratorio son, en general, inespecíficos; pero la mayoría de las series muestran una discreta elevación de la PCR y leucocitosis moderada o paraclínicos normales $(41-68 \%)$ [5, 13].

En general, el electrocardiograma es normal; pero algunos pacientes pueden presentar una amplia variedad de anormalidades que orienten a diagnósticos diferenciales, como bajo voltaje, taquicardia sinusal, desviación del eje, alternancia eléctrica, inversión de la onda $\mathrm{T}$, pérdida de la progresión de la onda $\mathrm{R}$, patrón S1Q3T3 y anormalidades del segmento ST, lo que en ocasiones puede desviar nuestra atención y orientar diagnósticos erróneos. Sin embargo, estos hallazgos desaparecen al resolver el neumomediastino y no se presenta el desarrollo de onda $Q$, relacionada con síndrome coronario agudo; tampoco hay elevación de biomarcadores que nos hagan sospechar esta patología [5, 20, 21].

La recurrencia del neumomediastino espontáneo es excepcional, y los datos en la literatura reportan muy pocos casos, sin lograr estimar cuál fue la causa de la recurrencia y cuánto es el tiempo libre de síntomas entre los dos episodios [5, 22, 23]. En la figura 1 se presenta el algoritmo diagnóstico.

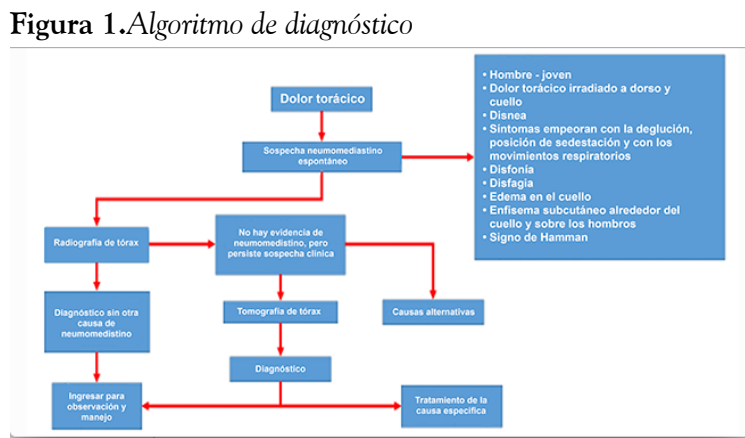

Fuente: Modificado de Sahni S, et al. Spontaneous Pneumomediastinum: Time for consensus. N Am J Med Sci. 2013;5(8):460-4.

\section{Manejo}

Una vez se han descartado las causas de neumomediastino secundario y que se asume que este es espontáneo, el tratamiento consiste en reposo, analgesia y en evitar las maniobras de Valsalva. En general, el tratamiento es expectante, dada la baja recurrencia [2, 3].

Habitualmente, el neumomediastino espontáneo es una patología benigna, con un curso limitado en el tiempo y una duración que varía desde 1,8 a 2,2 e, incluso, hasta una semana, según los diferentes reportes. La mayoría de los pacientes ingresados al hospital son manejados con analgésicos, reposo, oxígeno, broncodilatadores (cuando existe una patología concomitante asociada) y, ocasionalmente, tratamiento antibiótico.

El oxígeno suplementario aumenta la presión de difusión del nitrógeno en el intersticio, lo que promueve una rápida absorción del aire. Se debe realizar seguimiento imagenológico ambulatorio de los pacientes hasta evidenciar la resolución completa del neumomediastino, bien sea por radiografía de tórax simple o mediante el uso de tomografía (la literatura no describe el intervalo en el cual deben realizarse estas intervenciones) [7]. El uso de manejo antibiótico puede requerirse hasta en un $26 \%$ de los pacientes en quienes se asocia mediastinitis o septicemia relacionada principalmente con ruptura esofágica $[4,6]$. Los criterios de ingreso hospitalario abarcan 
la presencia de hipoxia, dificultad respiratoria, evidencia de perforación esofágica y necesidad de realización de procedimiento quirúrgico $[1,3,6$, 24].

\section{Complicaciones}

Es poco frecuente encontrar complicaciones relacionadas con el síndrome de Hamman; sin embargo, la demora en el diagnóstico y tratamiento puede llevar a una progresión del enfisema (pericardio, espacio pleural, espacio retroperitoneal y espacio retrofaríngeo) con la consecuente formación de neumotórax a tensión $-6 \%$ a $11 \%$ en algunas series, pero en el estudio desarrollado en la Clínica Mayo se presentó neumotórax asociado hasta en un $32 \%$ de los pacientes, de severidad variable y relacionada principalmente con patologías asociadas subyacentes del parénquima pulmonar - $[4,7]$, que lleva a la compresión de grandes vasos y bajo gasto cardiaco.

Para el caso específico de neumomediastino espontáneo, hasta ahora no se han reportado casos directos de mortalidad secundaria a dicha patología, y esta se relaciona con complicaciones de enfermedades subyacentes que acompañen el desarrollo de neumomediastino secundario hasta en un $8 \%$ [4]. Otras complicaciones incluyen compresión laríngea, seudotaponamiento cardiaco y mediastinitis en el contexto de perforación esofágica [25].

\section{Conclusiones}

El neumomediastino espontáneo es una condición benigna, poco común, de curso limitado y que afecta principalmente a hombres jóvenes. La sospecha diagnóstica en el clínico es fundamental para la evaluación del paciente en la indagación de síntomas específicos al interrogatorio, como disnea y dolor torácico, siendo este último el más común; así como la realización de un examen físico dirigido a la búsqueda de enfisema subcutáneo en el cuello y en la región supraclavicular, que orientan la toma de imágenes diagnósticas compatibles con neumomediastino.

Esta patología debe incluirse en los diagnósticos diferenciales en varones jóvenes que consulten por dolor torácico. La mayoría de los casos evolucionan satisfactoriamente con tratamiento sintomático, por lo cual es muy poco frecuente la recidiva. Las complicaciones más encontradas en la literatura están relacionadas con la presencia de neumotórax, que puede variar desde un $6 \%$ a un $11 \%$.

\section{Referencias}

1. Okada M, Adachi H, Shibuya Y, Ishikawa S, Hamabe Y. Diagnosis and treatment of patients with spontaneous pneumomediastinum. Respir Investig. 2014;52(1):36-40. doi: http://dx.doi.org/10 $.1016 / j . r e s i n v .2013 .06 .001$

2. Allen CJ, Teisch LF, Stahl KD. Spontaneous pneumomediastinum, pneumopericardium and epidural pneumatosis: insights on clinical management. Acute Card Care [Internet]. 2015;17(1):20-3. Disponible en: http://www.tandfonline.com/doi/full/10 .3109/17482941.2015.1005103

3. Ruiz-Ruiz F, Sampériz A, Rubio T, Escolar F. Neumomediastino espontáneo. An Sist Sanit Navar. 2006;29(2):275-8.

4. Iyer VN, Joshi AY, Ryu JH. Spontaneous pneumomediastinum: analysis of 62 consecutive adult patients. Mayo Clin Proc [Internet]. 2009;84(5):417-21. Disponible en: http://www.pubmedcentral.nih.gov/arti clerender.fcgi? artid $=2676124 \&$ tool $=$ pmc entrez\&rendertype $=$ abstract

5. Silva A, Barros D, Raddatz A. Neumomediastino espontáneo (síndrome de Hamman): una entidad poco frecuente no siempre reconocida. Rev Chil Cir. 2013;65(5):442-7.

6. Kim KS, Jeon HW, Moon Y, Kim Y Du, Ahn MI, Park JK, et al. Clinical experience of spontaneous pneumomediastinum: Diagnosis and treatment. J Thorac Dis. 2015;7(10):1817-24. 
7. Sahni S, Verma S, Grullon J, Esquire A, Patel P, Talwar A. Spontaneous pneumomediastinum: Time for consensus. N Am J Med Sci. 2013;5(8):460-4.

8. Bakhos CT, Pupovac SS, Ata A, Fantauzzi JP, Fabian T. Spontaneous pneumomediastinum: An extensive workup is not required. J Am Coll Surg. 2014;219(4):713-7. doi: http://dx.doi.org/1 0.1016/j.jamcollsurg.2014.06.001

9. Caceres M, Ali SZ, Braud R, Weiman D, Garrett HE. Spontaneous pneumomediastinum: a comparative study and review of the literature. Ann Thorac Surg [Internet]. 2008;86(3):962-6. Disponible en: http://www.ncbi.nlm.nih.go v/pubmed/18721592

10. Banki F, Estrera AL, Harrison RG, Miller CC, Leake SS, Mitchell KG, et al. Pneumomediastinum: Etiology and a guide to diagnosis and treatment. Am J Surg. 2013;206(6):1001-6. doi: http://dx.doi.org/ 10.1016/j.amjsurg.2013.08.009

11. Kim $\mathrm{H}$, Cho $\mathrm{T}$. Unusual presentation of spontaneous pneumomediastinum. Lung India [Internet]. 2010;27(4):239. Disponible en: http:/www.lungindia.com/t ext.asp?2010/27/4/239/71961

12. Bolvardi E, Pishbin E, Ebrahimi M, Mahmoudi Gharaee A, Bagherian F. Spontaneous pneumomediastinum with a rare presentation. Case Rep Emerg Med. 2014;2014(Figure 1):451407.

13. Kelly S, Hughes S, Nixon S, Paterson-Brown S. Spontaneous pneumomediastinum (Hamman's syndrome). Surgeon. 2010;8(2):63-6. doi: http://dx.doi.org/10.1 016/j.surge.2009.10.007

14. Kim SH, Huh J, Song J, Kang IS. Spontaneous pneumomediastinum: A rare disease associated with chest pain in adolescents. Yonsei Med J. 2015;56(5):1437-42.

15. Fitzwater JW, Silva NN, Knight CG, Malvezzi L, Ramos-Irizarry C, Burnweit CA. Management of spontaneous pneumomediastinum in children. J Pediatr Surg. 2015;50(6):983-6. doi: http://dx.doi. org/10.1016/j.jpedsurg.2015.03.024
16. Lee C-Y, Wu C-C, Lin C-Y. Etiologies of spontaneous pneumomediastinum in children of different ages. Pediatr Neonatol [Internet]. 2009;50(5):190-5. Disponible en: http://linkinghub.elsevier.com/retrieve/ pii/S1875957209600620

17. Gupta A, Rizvi I, Srivastava VK, Debnath J, Kapur BN, Khanna VN, et al. Spontaneous pneumomediastinum. Med J Armed Forces India [Internet]. 2015;71:S85-8. Disponible en: http://linkinghub.elsevier.co m/retrieve/pii/S0377123713000464

18. Zaia BE, Wheeler S. Pneumomediastinum after inhalation of helium gas from party balloons. J Emerg Med. 2010;38(2):155-8. Disponible en: http://dx.doi.org/10.1016/j.j emermed.2007.02.066

19. Lee SY, Sheu CY, Wu CL, Hung CL, Weng YL, Lin CC, et al. Spontaneous pneumomediastinum: A clinical radiologic analysis. Int J Gerontol. 2008;2(4):222-8. doi: http://dx.doi.org/10.1016/S1873-9598 (09) 70011-0

20. Kis A, Sutto Z, Tamasi L, Eszes $\mathrm{N}$, Losonczy G, Mathe $Z$, et al. Spontaneous pneumomediastinum after kidney transplantation: Case report. Transplant Proc. 2010;42(6):2350-2 . doi: http://dx.doi.org/10.1016/j.transprocee d.2010.05.016

21. Brearley WD, Taylor L, Haley MW, Littmann L. Pneumomediastinum mimicking acute ST-segment elevation myocardial infarction. Int $\mathrm{J}$ Cardiol. 2007;117(2):73-5.

22. Natale C, D'Journo XB, Duconseil P, Thomas PA. Recurrent spontaneous pneumomediastinum in an adult. Eur J Cardio-thoracic Surg. 2012;41(5):1199-201.

23. Jougon JB, Ballester $M$, Bride $T$ Mac, Dromer CEH, Velly JF. Assessment of spontaneous pneumomediastinum: experience with 12 patients. Ann Thorac Surg. 2003;75(6):1711-4.

24. Johnson NN, Toledo A, Endom EE. Pneumothorax, pneumomediastinum, and pulmonary embolism. Pediatr Clin North 
Am. 2010;57(6):1357-83. doi: http://dx.do i.org/10.1016/j.pcl.2010.09.009

25. Lee SC, Lee DH, Kim GJ. Is primary spontaneous pneumomediastinum a truly benign entity? Emerg Med Australas. 2014;26(6):573-8. 